\title{
Young star cluster complexes in NGC 4038/39^
}

\section{Integral field spectroscopy using VIMOS-VLT}

\author{
N. Bastian ${ }^{1,2, \star \star}$, E. Emsellem ${ }^{3}$, M. Kissler-Patig ${ }^{2}$, and C. Maraston ${ }^{4}$ \\ 1 Astronomical Institute, Utrecht University, Princetonplein 5, 3584 CC Utrecht, The Netherlands \\ e-mail: bastian@star.ucl.ac.uk \\ 2 European Southern Observatory, Karl-Schwarzchild-Strasse 2, 85748 Garching b. München, Germany \\ 3 CRAL-Observatoire de Lyon, 9 avenue Charles André, 69561 Saint-Genis-Laval Cedex, France \\ 4 University of Oxford, Denys Wilkinson Building, Keble Road, Oxford, OX13RH, UK
}

Received 7 July 2005 / Accepted 8 September 2005

\section{ABSTRACT}

We present the first results of a survey to obtain Integral Field Spectroscopy of merging galaxies along the Toomre Sequence. In the present work, we concentrate on the star cluster complexes in the Antennae galaxies (NGC 4038/39) in the overlap region as well as the nuclear region of NGC 4038. Using optical spectroscopy we derive the extinction, age, metallicity, velocity, velocity dispersion of the gas and star formation rate for each of the eight complexes detected. We supplement this study with archival $H S T$-WFPC2 $U, B, V, \mathrm{H} \alpha$, and $I$ band imaging. Correcting the observed colours of the star clusters within the complexes for extinction, measured through our optical spectra, we compare the clusters with simple stellar population models, with which we find an excellent agreement, and hence proceed to derive the ages and masses of the clusters from comparison with the models. In five of the complexes we detect strong Wolf-Rayet emission features, indicating young ages (3-5 Myr). The ionized gas surrounding the complexes is expanding at speeds of $20-40 \mathrm{~km} \mathrm{~s}^{-1}$. This slow expansion can be understood as a bubble, caused by the stellar winds and supernovae within the complexes, expanding into the remnant of the progenitor giant molecular cloud. We also find that the complexes themselves are grouped, at about the largest scale of which young star clusters are correlated, representing the largest coherent star forming region. We show that the area normalized star formation rates of these complexes clearly place them in the regime of star forming regions in starburst galaxies, thereby justifying the label of localized starbursts. Finally, we estimate the stability of the complexes, and find that they will probably loose a large fraction of their mass to the surrounding environment, although the central regions may merge into a single large star cluster.

Key words. galaxies: star clusters - galaxies: interactions - galaxies: individual: NGC 4038

\section{Introduction}

Mergers of gas-rich spiral galaxies can produce vast amounts of young massive star clusters. Examples of these extraordinary systems are NGC 7252 (Miller et al. 1997; Schweizer \& Seitzer 1998), NGC 3921 (Schweizer et al. 1996), NGC 1316 (Goudfrooij et al. 2001) and NGC 3256 (Zepf et al. 1999), all of which harbour populations of globular cluster sized star clusters which formed concurrently with the merger. The closest, and most spectacular ongoing spiral-spiral merger is NGC 4038/39 (also known as The Antennae) which contains thousands of massive star clusters in the process of forming (Whitmore et al. 1999). Due to its proximity and high cluster

* Based on Observations at the Very Large Telescope of the European Southern Observatory, Paranal/Chile under Program 71.B-3011.

$\star \star$ Current address: Department of Physics and Astronomy, University College London, Gower Street, WC1E 6BT, London, UK. formation rate, the cluster population of NGC 4038/39 is an almost ideal target to study cluster formation in its various stages as well as the impact that the formation has on the surrounding ISM. Additionally, Whitmore et al. (1999), among others, have noted several "knots", or cluster complexes, within the disks of these galaxies, which are collections of many individual young star clusters.

Using a wealth of multi-wavelength data, Zhang et al. (2001) showed that the youngest star clusters in NGC 4038/39 are not isolated, but instead tend to be distributed in a clustered fashion themselves. This implies that clusters do not form in isolation, but as part of a larger hierarchy of structure formation, extending from stellar to kiloparsec scales (Elmegreen \& Efremov 1996). These complexes of star clusters are the subject of the present study.

The best studied cluster complex is a large complex in the spiral galaxy NGC 6946. This complex has a diameter of 
$600 \mathrm{pc}$ and a total mass estimated to be $\sim 10^{7} M_{\odot}$. The most massive star cluster within the complex has a mass of $\sim 10^{6} M_{\odot}$ and an age of $15 \mathrm{Myr}$ (Larsen et al. 2002). Additionally there are other smaller star clusters within the complex which have similar ages (Elmegreen et al. 2000). This complex seems to have undergone three distinct bursts in its star formation history, one between 20 and $30 \mathrm{Myr}$ ago, the second, including the most massive cluster, around $15 \mathrm{Myr}$ ago, and a third which began roughly 6 to $8 \mathrm{Myr}$ ago and is continuing until the present (Larsen et al. 2002). Despite the multiple star formation epochs there is no clear relation between the age of the star clusters and their locations within the complex.

Other known large complexes (with and without star clusters) include the Gould Belt in the Galaxy (e.g. Stothers \& Frogel 1974), a large stellar complex in M 83 (Camerón 2001), as well as a series of cluster complexes along the western spiral arm of M 51. These latter complexes seem to follow the same mass vs. radius relation as giant molecular clouds, which appears absent at the level of the individual star clusters (Bastian et al. 2005b). This suggests that these complexes are the direct results of fragmentation of single giant molecular clouds.

Kroupa (1998) and Fellhauer \& Kroupa (2002) have suggested that cluster complexes (what they call superclusters) host a significant amount of merging of clusters within them. In their simulations this merging results in a massive extended star cluster which shows a radial age and metallicity gradient. The authors suggest (Fellhauer \& Kroupa 2005) that this is a mechanism to form extremely massive star clusters, like those observed in the merger remnants, such as W3 in NGC 7252 whose dynamical mass is $\sim 8 \times 10^{7} M_{\odot}$ (Maraston et al. 2004). Although their model fails to reproduce the precise properties of W3 (they under-predict the velocity dispersion) this mechanism may still be valid for other massive clusters.

To better understand the nature of cluster and cluster complex formation, we have obtained integral field spectroscopy of two regions in the merging galaxies NGC 4038/39. The intrinsic benefit of integral field unit spectroscopy is that it removes the bias of a pre-determined axis of study, as is the case with classical long-slit spectroscopy. This spectroscopic data is then combined with archival HST-WFPC2 broad and narrow band imaging which is able to spatially resolve each complex into its individual cluster components. The spectroscopic data presented here is one part of a much larger survey of the relationship between young massive star clusters, the interstellar medium, and the background stellar populations in interacting galaxies.

In Sect. 2 we present both the spectroscopic and photometric observations. The spectral features are introduced in Sect. 3 and we exploit them in Sect. 4 to derive their extinctions and metallicities. Section 5 is dedicated to deriving the ages of the complexes through the individual clusters within the complexes as well as by Wolf-Rayet features when available. The interstellar matter within the complexes, the star formation rates, and expanding shells are the topic of Sect. 6. In Sect. 7 we discuss the formation of the complexes and their relation to giant molecular clouds. The stability of the complexes is the subject of Sect. 8 and in Sect. 9 we summarize the main results.

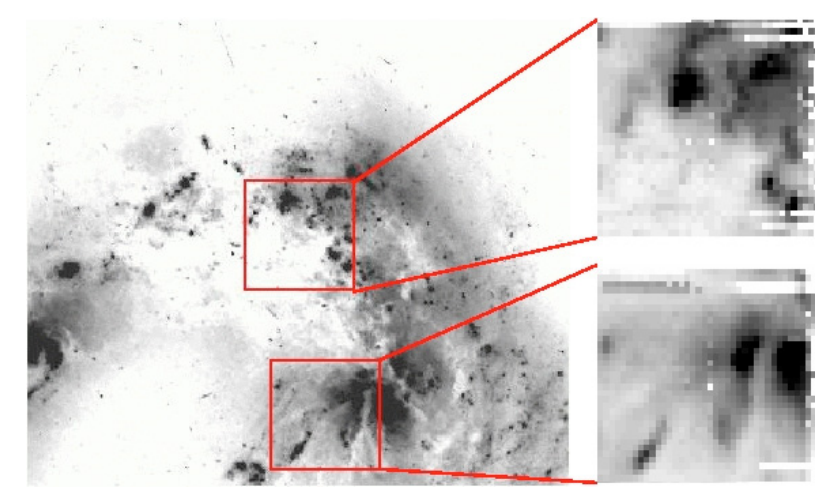

Fig. 1. Left: HST-WFPC2 F555W mosaic image of the Antennae. Right: the reconstructed VLT-VIMOS/IFU images shown in the same orientation, of the two pointings. Field 1 is shown on the top, while Field 2 is the bottom inset.

\section{Observations}

\subsection{VLT - VIMOS}

Two fields in the interacting galaxies NGC 4038/39, containing young massive star clusters, were observed with the VIMOS (Visual Multi-Object Spectrograph) Integral Field Unit on the $V L T$ during the nights of April 3rd, 4th, and 5th, 2003. The first field (hereafter Field 1) is located in the interaction region between the two galaxies (Region E + F, Fig. 6a in Whitmore et al. 1999), while the second field (hereafter Field 2) is centered on the nucleus of NGC 4038. The positions of the two fields are shown in Fig. 1. The observations were taken with the blue arm in high-resolution mode, covering the wavelength range $4150 \AA$ to $6100 \AA$ with a spectral resolution of $\sim 1.8 \AA$ at the position of $\mathrm{H} \beta$. The spatial sampling of the IFU was $0.66^{\prime \prime}$ covering $27^{\prime \prime}$ by $27^{\prime \prime}$ on the sky. Each individual exposure was $1200 \mathrm{~s}$ long, and we obtained 8 useful exposures resulting in a total exposure time for each field of $9600 \mathrm{~s}$. We also obtained off-target pointings in order to subtract the sky background. These are not used in the present work as we used neighbouring regions of the complexes to estimate the background, including the underlying contributions from both the sky and galaxies NGC 4038/39.

The data were reduced using the VIMOS/IFU pipeline ${ }^{1}$. The pipeline includes bias subtraction, flat-fielding, extraction of individual spectra, and wavelength calibration. The pipeline also produces reconstructed images of the field of view, which are shown in Fig. 1 (right). To obtain the spectra of each cluster complex, we summed the spectra from all the "spaxels" (spatial elements of the reconstructed image) containing flux from the cluster complex. The apertures were selected from the reconstructed images, and typically contained 12 to 18 spaxels. Due to the irregular apertures and the sizes of the complexes being comparable to the spatial resolution, no attempt was made to resolve the individual complexes. We then selected a region of background near each complex in order to extract a background spectrum, which was then subtracted from each cluster complex spectrum. These spectra were then flux-calibrated

\footnotetext{
1 The pipeline is available at http://www . eso.org/observing/ gasgano/vimos-pipe-recipes.html
} 


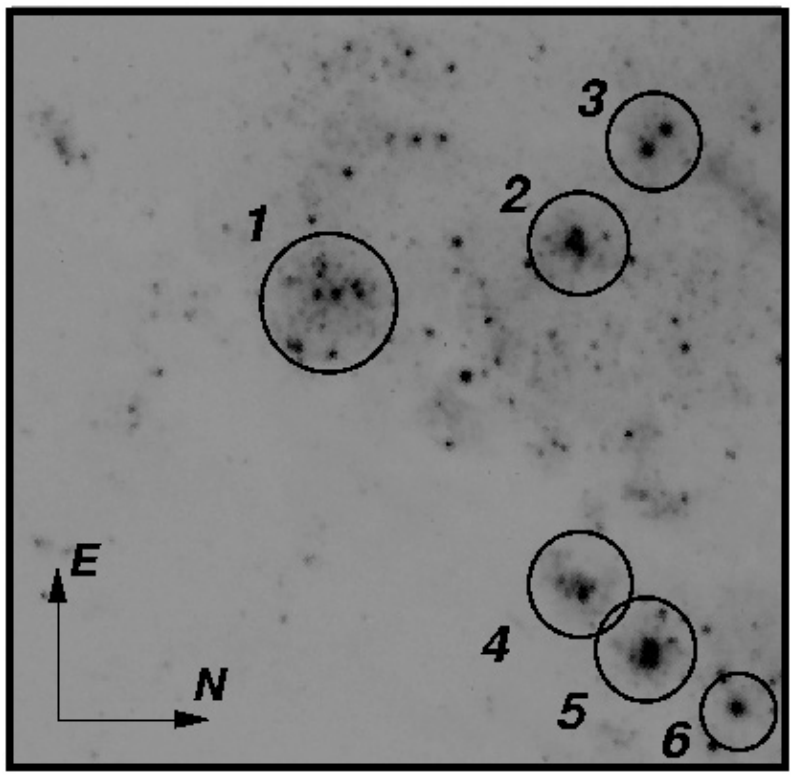

Fig. 2. Field 1. $H S T-W F P C 2 F 439 W$ image of the overlap region in NGC 4038. The image is $\sim 2.5 \mathrm{kpc}\left(27.5^{\prime \prime}\right)$ on a side. The complexes are identified and labelled Complex 1 through 6.

using photometric standard stars observed in each of the four VIMOS/IFU quadrants.

\subsection{HST - WFPC2}

We retrieved HST WFPC2 observations in the F336W $(U)$, $F 439 W(B), F 555 W(V), F 658 N(\mathrm{H} \alpha)$, and $F 814 W(I)$ filters from the $H S T$ archive. These data are presented in depth in Whitmore et al. (1999). Point-like sources were found using the DAOFIND task in IRAF and aperture photometry was carried out with the PHOT routine using an aperture, inner and outer background radii of 1.5, 3.5 and 5.5 respectively. CTE corrections were carried out using the formulae of Whitmore et al. (1999). Aperture corrections were determined from bright isolated sources. Comparison of our photometry to that of Whitmore et al. (1999) reveals that they are consistent within 0.03 in magnitude. Figures 2 and 3 show the F439W HST-WFPC2 images of Field 1 and Field 2 respectively. We adopt the same distance to the Antennae as Whitmore et al. (1999), namely 19.2 Mpc. At this distance, 1 arcsec corresponds to 93 pc so one wide field WFPC2 pixel and one VIMOS-IFU spaxel correspond to $9.3 \mathrm{pc}$ and $61 \mathrm{pc}$ repsectively.

The complexes in Field 1 are named Complex 1 through 6 , while the complexes in Field 2 are named Nuc 1 and Nuc 2 to designate them as belonging to the nuclear region of NGC 4038.

\section{Observed spectral properties}

\subsection{Introduction to the spectra}

The full spectra of each of the complexes are shown in Figs. 4 and 5. The dominating features of these spectra are the nebular emission lines $\mathrm{H} \gamma, \mathrm{H} \beta$, [O III] $(\lambda \lambda 4959,5007 \AA)$ and

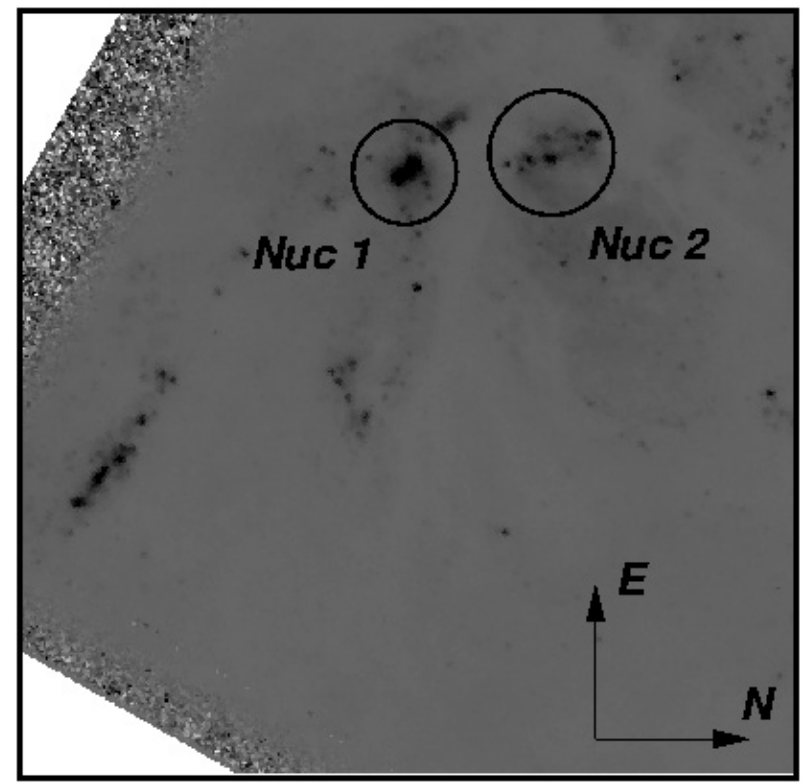

Fig. 3. Field 2. $F 439 W(B)$ band image of the nuclear region of NGC 4038. The two complexes in this region are labelled Nuc 1 and Nuc 2. Each side of the image is $\sim 2.5 \mathrm{kpc}\left(27.5^{\prime \prime}\right)$.

He I (15876), indicating the presence of a large amount of ionized gas. Additionally, Complexes 4, 5, Nuc 1, and Nuc 2 show strong Wolf-Rayet emission features (e.g. He II 14686), characteristic of extremely young stellar populations. Many of the complexes also show underlying stellar absorption features, the most notable example of this is Complex 3. All of these features will be discussed in detail in the following sections.

\subsection{Nebular emission line features}

Many of the spectra of the cluster complexes show a combination of (nebular) emission and underlying stellar absorption features. In the present study we are primarily interested in the emission features, namely $\mathrm{H} \gamma, \mathrm{H} \beta$, and [O III] $\lambda \lambda 4959,5007$ which can be used to estimate the extinction, metallicity, and total ionizing flux in the region. In order to remove the underlying absorption features from our data, we have employed the penalized pixel fitting method developed by Cappellari \& Emsellem (2004). This procedure fits a linear combination of template models (in this case the simple stellar population models) with the addition of a polynomial, to the observed spectra. We use the Vazdekis (1999) simple stellar population models as templates because these models have a spectral resolution $(\sim 1.8 \AA)$ that is comparable to that of the observed spectra. The best fitting combination template was then subtracted from the observed spectra, leaving a pure emission line spectrum. An example of this technique for two sections of the spectra of Complex 3 is shown in Fig. 6.

We then measured the wavelength, flux, and FWHM of each of the emission lines with the SPLOT routine in IRAF, approximating each line as a Gaussian. For the kinematic determination we measured the $\mathrm{H} \gamma, \mathrm{H} \beta$, [O III] $\lambda \lambda 4959,5007$, and HeII $(\lambda 5876)$ emission lines when available. Typical velocity errors are $\sim 5-10 \mathrm{~km} \mathrm{~s}^{-1}$, calculated as the standard deviation of 


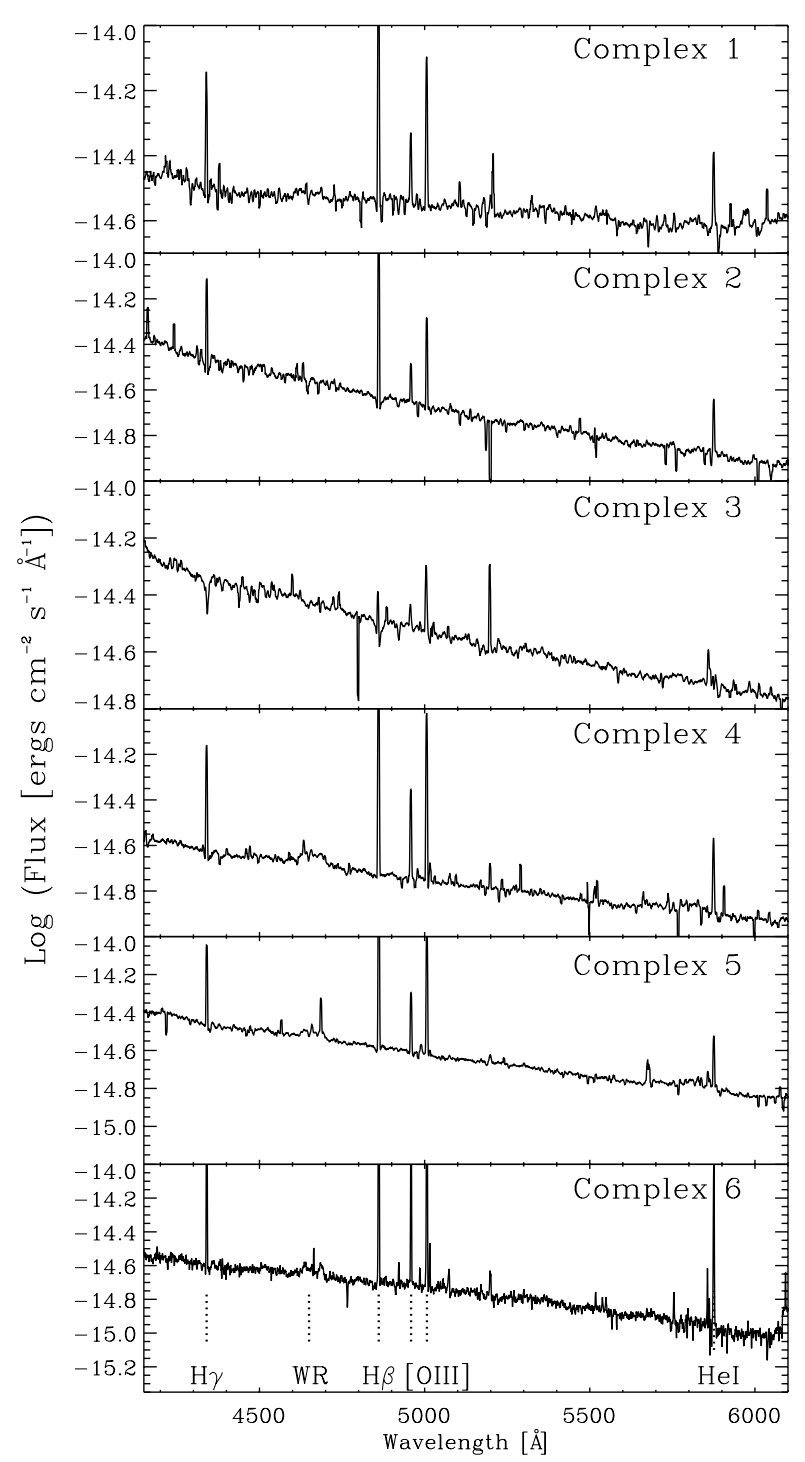

Fig. 4. Observed spectra of the cluster complexes in Field 1. All spectra are in the rest wavelength of NGC 4038, and have not been corrected for extinction. Prominent emission lines are indicated, including the Wolf-Rayet feature at $\sim 4650 \AA$. The spectra have been smoothed using a boxcar function with width $4.5 \AA$.

the velocities measured from each individual line. The derived extinctions and velocities are shown in Table 1 . We note that our Complexes 1 in Field 1 corresponds to Region E as well as Complexes 4 and 5 corresponding to Region F of Whitmore et al. (2005). Comparing the derived velocities between that work and ours shows that the measured velocities differ by less than $5 \mathrm{~km} \mathrm{~s}^{-1}$.

We do not detect [O III] $\lambda 4363$ in any of our spectra, thus we were not able to estimate the temperature of the ionized regions. Non-detection of this line suggests metallicities $>0.5(\mathrm{O} / \mathrm{H})_{\odot}($ Kennicutt et al. 2003). As will be seen in the next section, this is consistent with what we find through the comparison with stellar population models as well as from empirically calibrated emission line ratios, with all complexes studied here being nearly or above $0.5 Z_{\odot}$.

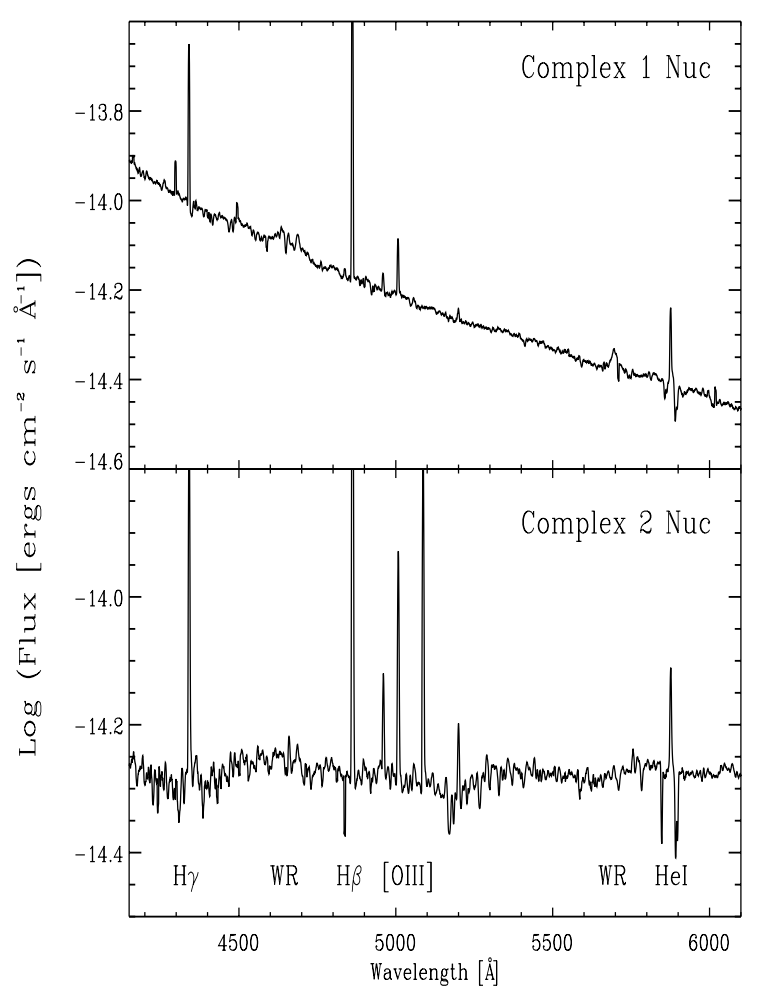

Fig. 5. Observed spectra of the cluster complexes in Field 2. All spectra are in the rest wavelength of NGC 4038, and have not been corrected for extinction. Prominent emission lines are indicated, including the Wolf-Rayet feature at $\sim 4650 \AA$ (the blue bump) as well as the Wolf-Rayet feature at $5696 \AA$ which is the broad emission from $\mathrm{C}$ III. The spectra have been binned using a boxcar function with width $4.5 \AA$.

\section{Extinction and metallicity of the complexes}

By measuring the relative strengths of $\mathrm{H} \gamma$ to $\mathrm{H} \beta$, we can estimate the amount of extinction within the complex. For Case $\mathrm{B}$ recombination at $10000 \mathrm{~K}$ the ratio of $\mathrm{H} \gamma$ to $\mathrm{H} \beta$ is 0.466 (Osterbrock 1989). By comparing the observed value of $\mathrm{H} \gamma / \mathrm{H} \beta$ and assuming the extinction curve of Savage \& Mathis (1979) we find extinctions, $A_{F 555 W}$, between 0 and $\sim 1.5$. Care must be taken when interpreting these numbers, however, as extinction most likely varies across each complex. Unfortunately our observations do not have the spatial resolution to correct for this effect. We therefore apply the average extinction estimate of each complex to each source within that complex. The one exception to this is Complex 1. The lack of $\mathrm{H} \alpha$ emission at the position of Complex 1 and the strong HII region nearby lead us to conclude that the emission lines and Wolf-Rayet features come from the neighboring HII region, and not from Complex 1 itself. Thus, we do not know the extinction for Complex 1 and we therefore assign the clusters within Complex 1 zero extinction.

We can also use the spectra to estimate the metallicity of each complex. As is the case with extinction, we do not have high enough spatial resolution to detect variations in metallicity across the complex. However, we do not expect large metallicity gradients within each complex as all sources within a complex presumably formed from the same giant molecular cloud or cloud complex. From Vacca \& Conti (1992), we derive the 
Table 1. Positional and general information on the observed complexes.

\begin{tabular}{ccccccccc}
\hline \hline ID $^{a}$ & RA (J2000) & Dec (J2000) & $M_{V}^{a}$ & $A_{V}$ & $\begin{array}{c}Z \\
\left(Z_{\odot}\right)\end{array}$ & $\begin{array}{c}V_{r}^{b} \\
\left(\mathrm{~km} \mathrm{~s}^{-1}\right)\end{array}$ & $\begin{array}{c}\sigma^{c} \\
\left(\mathrm{~km} \mathrm{~s}^{-1}\right)\end{array}$ & Notes \\
\hline $1^{d}$ & $12: 01: 55.60$ & $-18: 52: 21.8$ & -13.8 & 0.0 & 0.97 & $-58.5 \pm 13.8$ & 43 & weak WR features, contamination by close HII region \\
2 & $12: 01: 55.73$ & $-18: 52: 13.3$ & -13.6 & 0.0 & 1.3 & $-21.3 \pm 4.25$ & 33 & \\
$3 \mathrm{a}$ & $12: 01: 55.99$ & $-18: 52: 10.4$ & -13.6 & 0.0 & 0.45 & $-152.8 \pm 9.1$ & & \\
$3 \mathrm{~b}$ & & & & & & $-17.0 \pm 19.2$ & 73 & broad absorption feature superimposed on emission lines \\
4 & $12: 01: 54.86$ & $-18: 52: 12.9$ & -13.5 & 0.72 & 0.75 & $-51.7 \pm 7.5$ & 37 & strong WR features \\
5 & $12: 01: 54.71$ & $-18: 52: 10.6$ & -14.0 & 0.67 & 0.87 & $-6.0 \pm 6.1$ & 29 & strong WR features \\
6 & $12: 01: 54.58$ & $-18: 52: 07.2$ & -13.1 & 0.91 & 0.76 & $-3.5 \pm 5.7$ & 29 & strong WR features \\
1 Nuc & $12: 01: 52.97$ & $-18: 52: 08.4$ & -14.6 & 0.0 & $>2^{e}$ & $13.6 \pm 3.7$ & - & strong WR features \\
2 Nuc & $12: 01: 53.04$ & $-18: 52: 02.7$ & -14.3 & 1.0 & 1.67 & $60.5 \pm 21.0$ & - & WR features \\
\hline
\end{tabular}

$a$ Within the 15 pixel $(140 \mathrm{pc})$ radius used for source detection, uncorrected for extinction.

${ }^{b}$ Relative to the velocity of NGC 4038, taken to be $V=1642 \mathrm{~km} \mathrm{~s}^{-1}$.

$c$ The velocity dispersion of the gas, as measured from the $\mathrm{H} \beta$ and $\mathrm{O}[\mathrm{III}]$ lines.

${ }^{d}$ Measured extinction $\left(A_{\mathrm{V}}=1.3 \mathrm{mag}\right)$ and metallicity presumably refer to the contaminating HII region nearby, as these quantities are measured from emission lines. For the photometric analysis, no extinction correction has been applied.

e $R_{3}$ is out of the calibrated range.
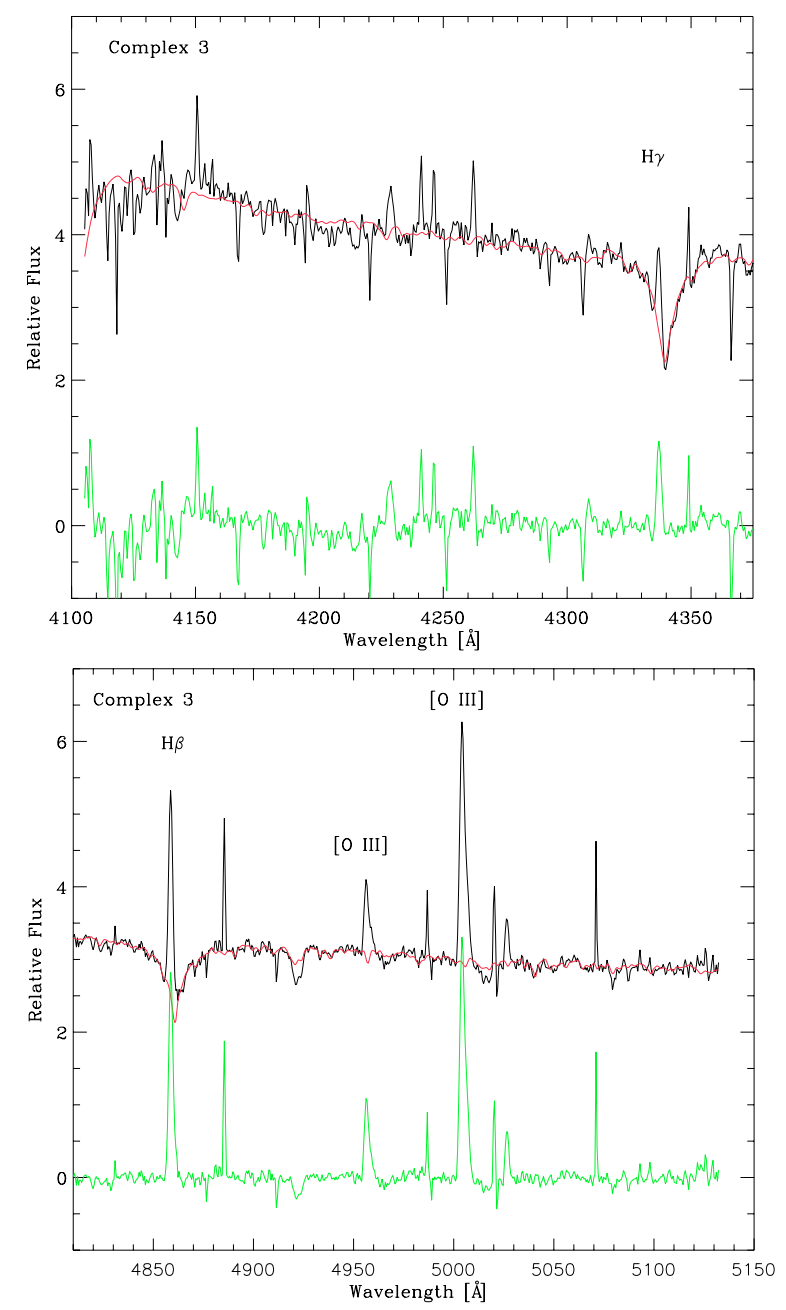

Fig. 6. Top: the blue region of the spectrum of Complex 3 in Field 1. The black line is the observed spectrum, the red line is the best fitting stellar template, and the green line is the pure emission line spectrum (the observed spectrum minus the stellar template). Bottom: the same as the top panel, but now for the red section of the spectrum of Complex 3.

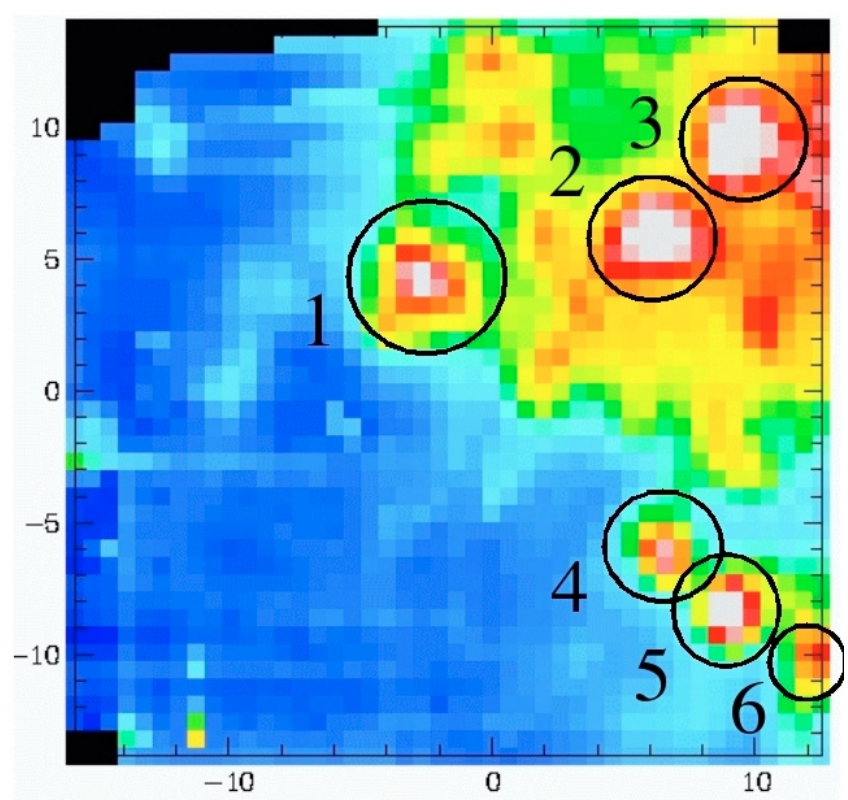

Fig. 7. Reconstructed image of the total intensity of the VIMOS-IFU observations of Field 1 with the five complexes labeled by number (the sixth is out of the field of view on many of the expssures so we will not study the spectrum in detail in the present work). The orientation is the same as in Fig. 2, and the image is $27^{\prime \prime}$ on a side ( 2.5 kpc).

$\mathrm{O} / \mathrm{H}$ ratio in the emission line regions using:

$\log (\mathrm{O} / \mathrm{H})=-0.69 \log R_{3}-3.24\left(-0.6 \leq \log R_{3} \leq 1.0\right)$

where

$R_{3}=\frac{I([\mathrm{O} \mathrm{III}] \lambda 4959)+I([\mathrm{O} \mathrm{III}] \lambda 5007)}{I(\mathrm{H} \beta)}$.

The estimated intrinsic uncertainty in the calibration of this method is \pm 0.2 in $\log (\mathrm{O} / \mathrm{H})$ (Edmunds \& Pagel 1984). We have adopted the "Local Galactic" oxygen abundance of $(\mathrm{O} / \mathrm{H})_{\odot}=$ $8.30 \times 10^{-4}$ (Meyer 1985) for the "solar" abundance. 


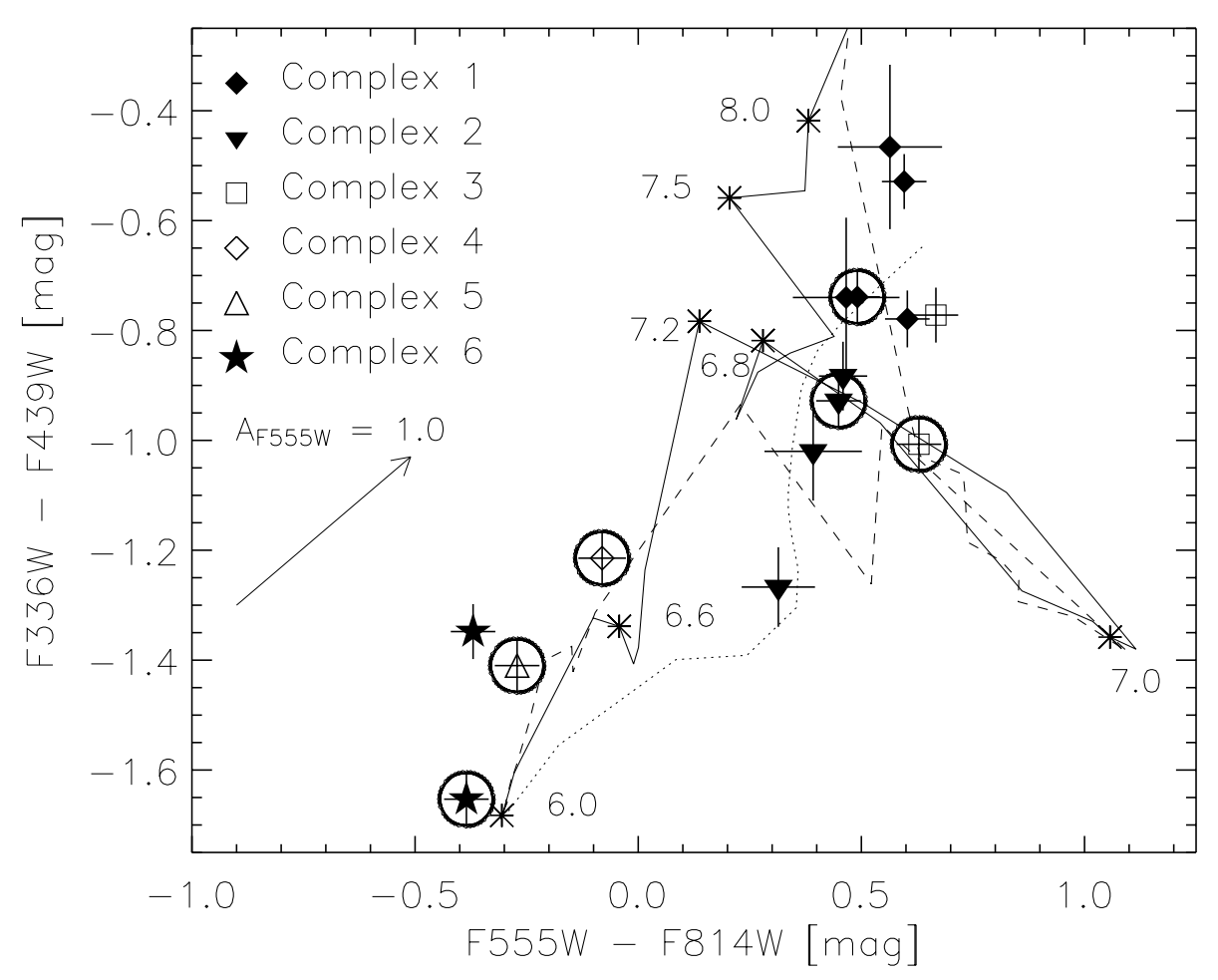

Fig. 8. Colour-colour diagram of the clusters within the five complexes in Field 1. The dashed and solid lines are the Maraston model (2005) tracks for solar and twice solar metallicity, respectively (the numbers correspond to the logarithm of the age in years at that point). The dotted line represents the changing colours of a model of continuous star formation of solar metallicity (Maraston 2005), with the final point representing an age of $1 \mathrm{Gyr}$. All points have been corrected for the average extinction of the complex (see text). The brightest cluster in each complex is circled. Sources within each complex have been labeled as follows; solid diamonds for Complex 1, filled triangles for Complex 2, open squares for Complex 3, open diamonds for Complex 4, and open triangles for Complex 5.
This empirical relation, however, has been shown to be affected by the hardness of the ionizing photons. Another empirical relation has been introduced which attempts to compensate for this effect, the $R_{23}$ relation. The relation includes the contribution from another ionized species of oxygen, namely [O II], which takes into account the ionization structure of oxygen. Unfortunately our wavelength range does not extend to the [O II] $\lambda 3727 \AA$ line, which is required for the $R_{23}$ calibration. Also see Kennicutt et al. (2003) for a discussion on the accuracy of using collisionally excited lines for metallicity determination.

Despite the above caveats, we list the measured metallicities of the complexes in Table 1.

\section{Ages of the cluster complexes}

\subsection{Ages of the individual clusters based on photometry}

A common technique employed to derive the ages of individual clusters is to compare the spectral energy distribution of each cluster to that of simple stellar population models (e.g. Bastian et al. 2005a). We use simple stellar population models (SSP) from Maraston (2005). At ages <30 Myr, these models are based on the tracks/isochrones from the Geneva group (Schaller et al. 1992; Schaller et al. 1993) and span the metallicity $\mathrm{Z} / \mathrm{H}$ range from $1 / 50$ to $2 Z_{\odot}$. The IMF can be Salpeter (1955) or Kroupa (2003), from 0.8 to $120 M_{\odot}$ (where we have chosen the Kroupa type IMF).

One of the main degeneracies in this kind of study, is between age and extinction for young clusters. This can be seen in Fig. 8 where the extinction vector is almost parallel to the SSP models for much of the first $\sim 10^{7.5}$ years of a cluster's existence. In the present study, however, we estimated the extinction independently, using the $\mathrm{H} \gamma / \mathrm{H} \beta$ ratio from our spectroscopy. Therefore, we have corrected the photometry of each cluster within the complexes for the extinction value derived in Sect. 4 (except Complex 1 as discussed above). It should be noted that the extinction for the individual clusters is not necessarily the same as the nebular extinction (as derived from the $\mathrm{H} \gamma / \mathrm{H} \beta$ ratio). However, if the emission lines are generated near the clusters and the majority of the extinction occurs outside this region, then the extinction of the clusters and the nebular extinction should be approximately equal. Throughout this work we will assume that this assumption holds.

Figures 8 and 9 show the colours of all clusters that have errors of less than 0.15 in their colours. The brightest cluster in each complex is circled. The Maraston (2005) SSP models of different metallicities, $Z_{\odot}, 2 Z_{\odot}$ are overplotted as solid and dashed lines respectively. The dotted line in Fig. 8 represents a solar metallicity continous star formation model. The numbers correspond to the log of the age of the models (in years), which are also marked with an asterisk.

The models do an excellent job in reproducing the observed colours, once extinction is removed. The masses of the complexes are derived by measuring the light of the complex (corrected for extinction) and comparing it with the mass-to-light ratios (including remnants) of the SSP models. Table 2 shows the derived ages and masses from the photometric method. The age (and hence the mass) of Complex 3 is poorly constrained as it is located near the "red loop" in colour space for solar metallicity SSP models.

The photometric ages of the brightest cluster in each complex derived in this section are shown in Table 2, and range from a few Myr to 25 Myr. We note that the relative ages of the brightest clusters in Complexes 4, 5, and 6, are consistent with the size of the HII region surrounding each of them, in 


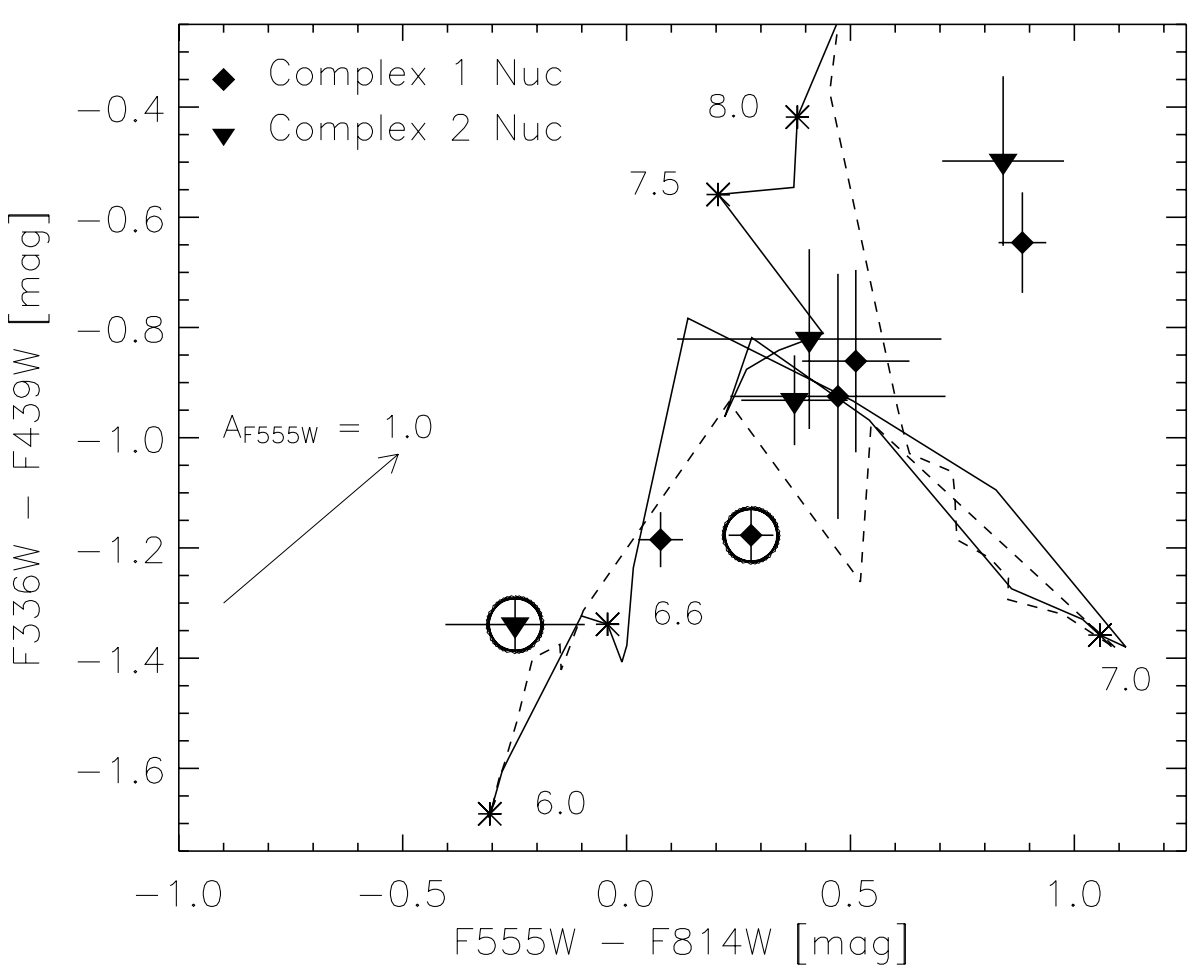

Fig. 9. Same as Fig. 8 but now for clusters inside the complexes in the nuclear region of NGC 4038 (Field 2). Filled diamonds are clusters within Complex Nuc 1 while filled triangles represent clusters in Complex Nuc 2. The two clusters from complex Nuc 1 near the lower left model tracks dominate the optical light from the complex. Complex Nuc 2 seems to have clusters within its projected radius with ages between a few Myr and a few tens of Myr, although we note that the clusters lie parallel to the extinction vector suggesting a similar age but variable extinction. The brightest cluster in each complex is circled.

Table 2. Derived properties of the complexes.

\begin{tabular}{ccccccccc}
\hline \hline ID & Field & $\begin{array}{c}\mathrm{Age}^{a} \\
(\mathrm{Myr})\end{array}$ & $\begin{array}{c}\mathrm{Age}^{b} \\
(\mathrm{Myr})\end{array}$ & $\begin{array}{c}\mathrm{Mass}^{c} \\
\left(10^{6} M_{\odot}\right)\end{array}$ & $M_{F 555 W}^{d}$ & $\begin{array}{c}R_{F 555 W}^{e} \\
(\mathrm{pc})\end{array}$ & $\begin{array}{c}R_{\mathrm{H} \alpha}^{e} \\
(\mathrm{pc})\end{array}$ & $\begin{array}{c}S F R^{f} \\
\left(M_{\odot} / \mathrm{yr}\right)\end{array}$ \\
\hline 1 & 1 & $20-30$ & - & $2.0-2.5$ & -11.50 & - & - & 1.40 \\
2 & 1 & $6-16$ & - & $0.4-1.3$ & -11.23 & 198 & 94 & 0.20 \\
3 & 1 & $7-14$ & - & $0.4-1.2$ & -12.31 & 200 & - & - \\
4 & 1 & $3.5-5.5$ & $3-4.5$ & $0.7-1.0$ & -12.73 & 136 & 180 & 0.43 \\
5 & 1 & $3-4$ & $2.5-3$ & $1.1-1.2$ & -13.15 & 175 & 189 & 0.35 \\
6 & 1 & $1-2$ & $2.5-3$ & $0.6-1.2$ & -13.03 & 90 & 100 & - \\
$1 \mathrm{Nuc}$ & 2 & $4-6$ & $3.5-4.5$ & 0.9 & -13.49 & 123 & 238 & 0.47 \\
$2 \mathrm{Nuc}$ & 2 & 3.5 & $3.5-4.5$ & 1.1 & -12.12 & - & - & 0.70 \\
\hline
\end{tabular}

a The age determined from the comparison of the photometry of the brightest cluster to that of SSP models of solar metallicity.

$b$ The age determined from Wolf-Rayet emission features.

$c$ The mass of the complex, corrected for extinction, using the M/L ratio of the Maraston (20055) SSPs (solar metallicity) and the age derived from the colours of the brightest star cluster in the complex.

$d$ The magnitude of the brightest cluster within the complex, corrected for extinction.

$e$ The size (diameter) of the complex as measured in the $F 555 \mathrm{~W}$ and the $\mathrm{H} \alpha$ filters.

$f$ Based on the $\mathrm{H} \beta$ emission flux.

the sense that the youngest complex (Complex 6) has a smaller HII region surrounding it. Presumably, the bubble caused by the winds and supernovae inside this complex has not had enough time to expand to a larger extent. We will return to this point in Sect. 6.2.

From the current data (see Figs. 8 and 9) we cannot rule out the existence of clusters with different ages $(\Delta(\mathrm{age}) \geq 5 \mathrm{Myr})$ within the same complex. This is due to the colours of different clusters lying parellel to the extinction vector. Spectroscopy of individual clusters is required to derive the extinction of each individual cluster in order to break the age/extinction degeneracy. We may expect an age difference within a single complex based on the works of Elmegreen et al. (2000) and Larsen et al. (2002) who found clusters with different ages in the large cluster complex in NGC $6946(\Delta($ age $) \approx 30 \mathrm{Myr})$. Despite the possible presence of these multi-age populations, we assign the age of the brightest cluster to that of the complex for further comparison.

We can most clearly see the caveat of our extinction determination in Complex Nuc 2 in Fig. 9. There, we see that all the clusters within the complex can be connected by the extinction arrow in colour space. Thus, these clusters could be older, low extinction objects, or younger, high extinction objects. So we can only conclude that there is either a large difference in 


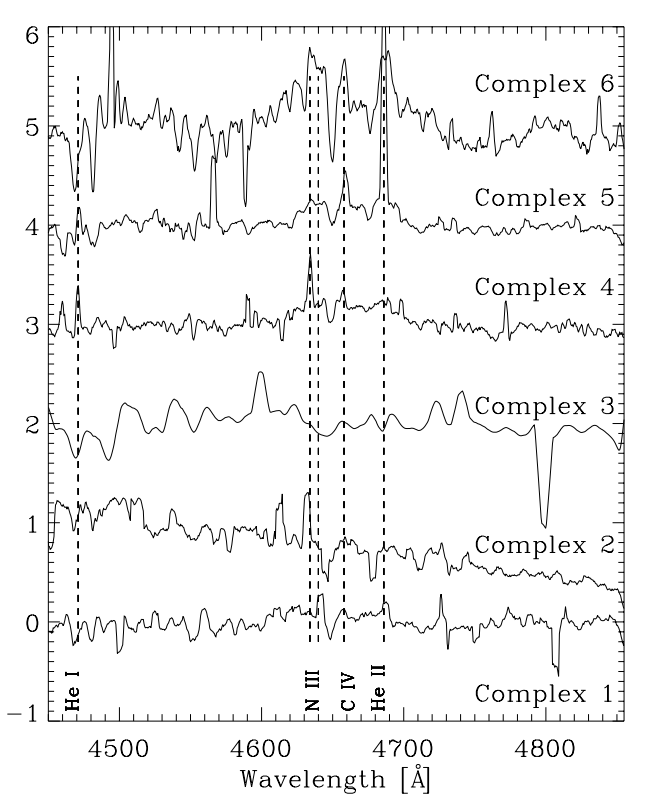

Fig. 10. The region of the spectrum around the "blue bump" WolfRayet ( $\sim 4650 \AA$ A, made up of the N III, C IV and He II broad emmision lines) feature for the five complexes in Field 1. The continuum has been subtracted from each spectrum. The WR feature is clearly observed in Complexes 4 and 5, and weakly, though definitely present, in Complex 1.

reddening across the complex or that there exists a multiple age population. Spectroscopy of individual complex members is required to break this degeneracy.

\subsection{Age dating of the complexes based on their Wolf-Rayet features}

We can take advantage of the presence of young massive stars within each complex by using their Wolf-Rayet features to independently derive their ages. Wolf-Rayet stars produce strong broad emission lines which can be identified in the composite spectrum of a stellar population. This technique has the advantage that it is independent of extinction and can be used to verify the ages determined through their colours. The spectral region around the "blue bump" Wolf-Rayet feature ( $\lambda 4650 \AA$ ) for each of the complexes is shown in Figs. 10 and 11.

The spatial resolution of the observations allow us to find where strong Wolf-Rayet features are present within the field of view. To do this, we fit a polynomial to the spectra region around the "blue bump" (see Fig. 10), and then subtract the continuum. We then summed the remaining flux in that region, and reconstruct the spatial image. The results of this are shown in Fig. 12 where bright colours represent strong WolfRayet features and dark colours represent regions where little or no features are detected. Here we see that Complexes 4, 5, and 6 (Complex 6 being mostly off the image however) are the only regions of strong WR features in this field. From the fact that these three complexes show WR features, we can conclude that they (or at least the massive stars within the complex) have the same age within $4 \mathrm{Myr}$ (Norris 2003), and all have ages less than 10 Myr. This strongly suggests that the

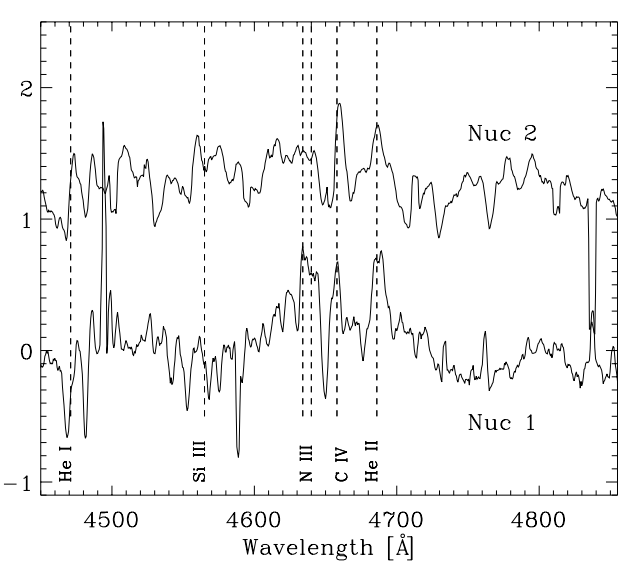

Fig. 11. Same as Fig. 10 but now for the two complexes present in the nuclear region of NGC 4038. Complex Nuc 1 has a strong WR feature, while in Nuc 2 the feature is either weak or absent.

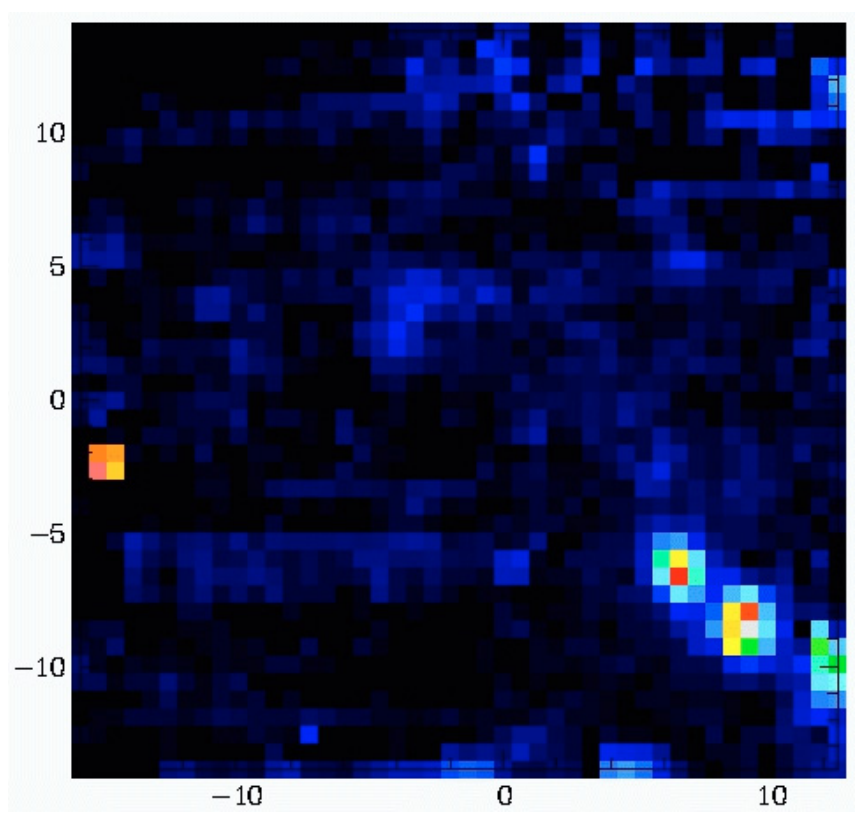

Fig. 12. The spatial distribution of Wolf-Rayet features in Field 1. Brighter colours indicates strong Wolf-Rayet features. Note that Complexes 4, 5, 6 all show strong WR features. The orientation is the same as in Fig. 2. One spaxel (spatial pixel) in this image is $63 \mathrm{pc}$.

formation of these complexes, or at least the most recent round of star formation within them, was triggered at about the same time. We shall return to this point in Sect. 7. We also point out that Complexes 4, 5, and 6 do not appear to have any old clusters within them (see Fig. 8) suggesting that the most recently round of star formation is the only significant period of star formation to take place within these complexes. While we cannot rule out the presence of an older population of stars within the complexes (age $>100 \mathrm{Myr}$ ), we do note that such a population would not affect colours, and hence the derived ages, of the detected clusters in these complexes due to the fact that young clusters significantly outshine their older counterparts in the optical wavelengths.

Finally, we note that the clusters in Complexes 4, 5, and 6 lie far from the continuous star formation models (in colour space) and much closer to the SSP model colours, whereas the 


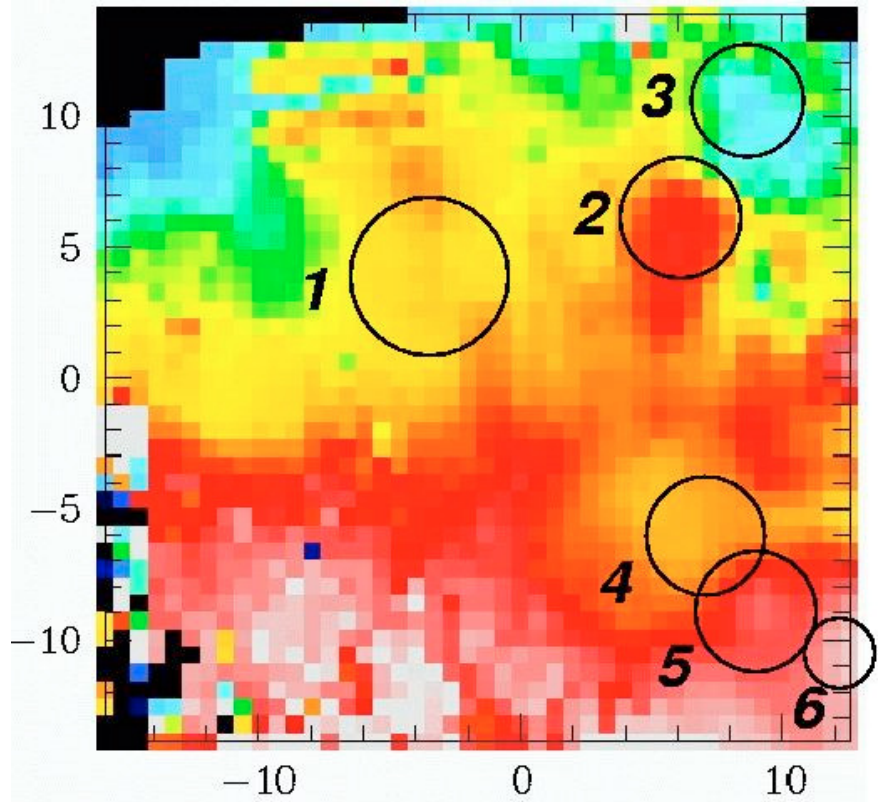

Fig. 13. The velocity field of Field 1 . The scale runs from $1400 \mathrm{~km} \mathrm{~s}^{-1}$ (blue) to $1650 \mathrm{~km} \mathrm{~s}^{-1}$ (white). One spaxel (spatial pixel) in this image is $63 \mathrm{pc}$.

clusters in Complex 2 are also compatible with the continous cluster formation models (the dotted line in Fig. 8).

\section{Interstellar matter in the cluster complexes}

\subsection{General velocity field}

Figure 13 shows the velocity field of the gas (fit on both the $\mathrm{H} \beta$ and [OIII] $\lambda \lambda 44959,5007 \AA$ absorption lines) in Field 1. The complexes and their positions are indicated. The scale runs from $1400 \mathrm{~km} \mathrm{~s}^{-1}$ (blue) to $1650 \mathrm{~km} \mathrm{~s}^{-1}$ (white). In general we see that the velocity changes from higher to lower from the bottom of the image to the top. This is in good agreement with the general velocity pattern of the Antennae in this region, i.e. as measured in HI (Hibbard et al. 2001).

\subsection{Expansion velocities}

In order to estimate the velocity dispersion of the gas (a measure of the random motions of the gas), we have fit the width of the $\mathrm{H} \beta$ and [O III] $\lambda \lambda 4959,5007$ emission lines after subtraction of the best-fitting stellar background template. To correct for the instrumental resolution, we also measured the sky line at $\lambda 5577$ and subtracted the width of this line in quadrature for the $\mathrm{H} \beta /[\mathrm{O}$ III $]$ combination. This routine was carried out for every spectrum (i.e. for every spaxel) in our data cube. The resulting velocity dispersion map for Field 1 is shown in Fig. 14, where dark blue represents the regions with the lowest velocity dispersion and white represents regions with the highest dispersion (the scale runs from 0 to $100 \mathrm{~km} \mathrm{~s}^{-1}$ ). The average value for each complexe is shown in Table 1.

The complexes (marked with circles and labeled in the figure) and the regions around them, show small velocity dispersions, of the order of $20-40 \mathrm{~km} \mathrm{~s}^{-1}$. These values can be

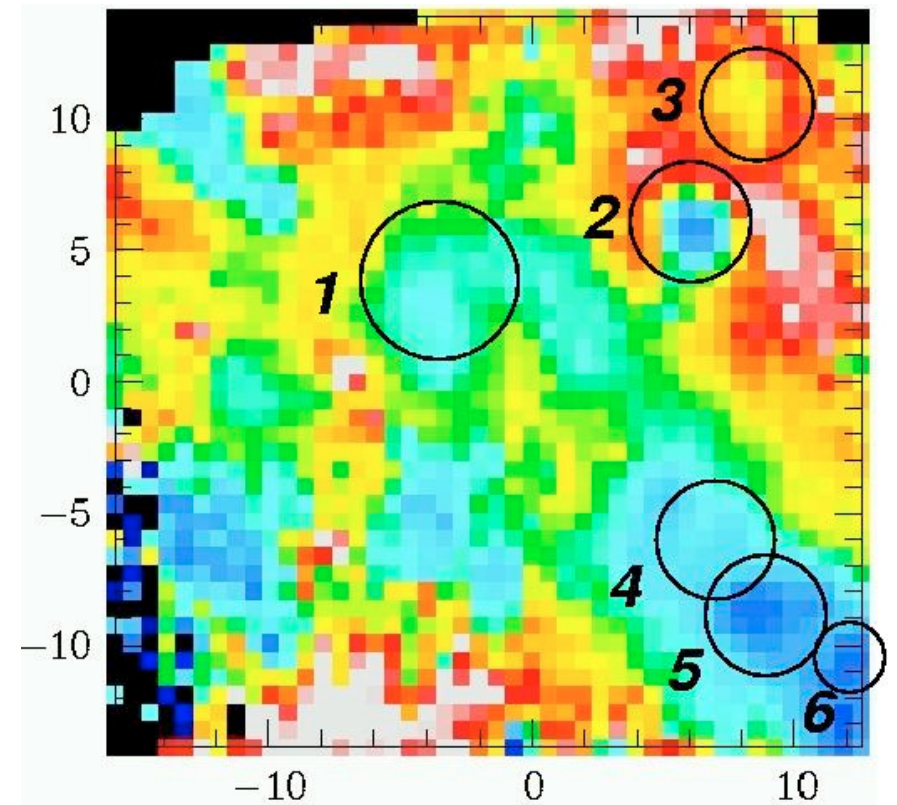

Fig. 14. The velocity dispersion of the gas as measured from the combination of $\mathrm{H} \beta$ and the [O III] $\lambda \lambda 4959,5007$ lines. The colour coding extends from 0 to $100 \mathrm{~km} \mathrm{~s}^{-1}$, with blue representing the lowest velocity dispersions, and white representing the highest. The measurements have been corrected by subtracting in quadrature the measured width of the skyline at $\lambda 5577$. Note that the gas at the position of the complexes has the lowest velocity dispersion in the region $\left(20-40 \mathrm{~km} \mathrm{~s}^{-1}\right)$. One spaxel (spatial pixel) in this image is $63 \mathrm{pc}$.

understood as bubbles, originating from a cluster wind, which is made up from the collection of winds from hot stars and supernovae. If one assumes that the winds/SNe act collectively to form a cluster wind, then one can estimate the speed of expansion of the resulting bubble using equations 12.12 and 12.19 in Lamers \& Cassinelli (1999). For this we assume that the ambient medium that the bubble is expanding into has a typical density of a GMC in the Antennae $\left(0.3 M_{\odot} / \mathrm{pc}^{3}-\right.$ Wilson et al. 2003) and a wind kinetic luminosity of between $10^{39}$ and $10^{42} \mathrm{erg} / \mathrm{s}$ (estimated from Tenorio-Tagle et al. 2005). We further assume that the wind has been blowing constantly for the past 3-7 Myr. With these assumptions (and assuming that the driven bubble is in the energy or momentum "snowplow" phase) the bubble outflow velocity is expected to be between 10 and $35 \mathrm{~km} \mathrm{~s}^{-1}$. These values are in excellent agreement with the observed velocity dispersions.

The exception to the above argument is Complex 3, which has a much higher velocity dispersion than the other complexes. However, we do not detect strong $\mathrm{H} \alpha$ or $\mathrm{H} \beta$ emission associated with this complex (the sources within Complex 3 also appear older than $10 \mathrm{Myr}$ based on their colours), hence the velocity dispersion that we measure is likely to be associated with the background.

\subsection{Star formation rates}

We can also estimate the star formation rates of the complexes. Our optical spectra do not extend to the $\mathrm{H} \alpha$ emission line, therefore we will use the dereddened $\mathrm{H} \beta$ line. To do this, we 

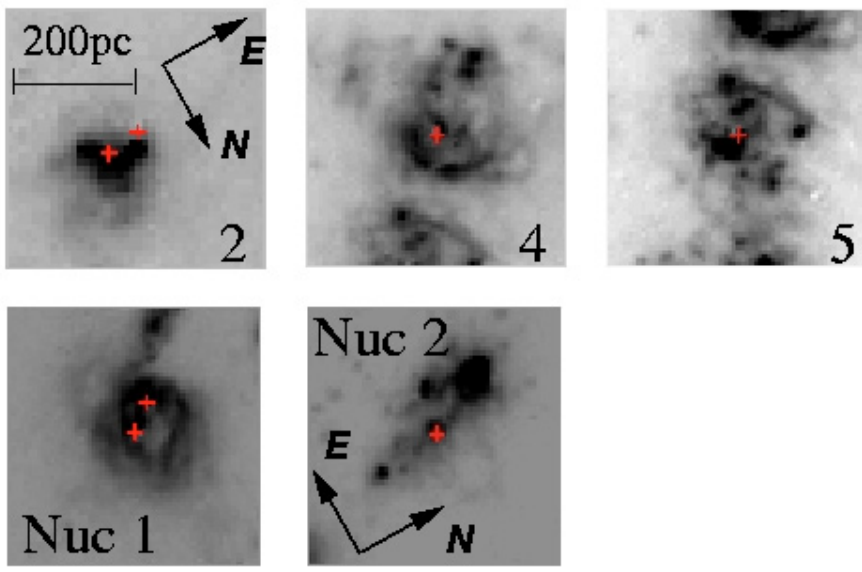

Fig. 15. The positions of the brightest cluster within each complex, superimposed on a continuum subtracted $\mathrm{H} \alpha$ image. The complex IDs are given in each panel. Each image is centered on the brightest source, other sources shown are other young clusters in the complex as seen from Figs. 8 and 9, presumably responsible for creating the surrounding HII regions. All panels have the same scale, Complexes 2, 4 and 5 share the same orientation, while Nuc 1 and Nuc 2 also have the same orientation.

measure the flux of the $\mathrm{H} \beta$ line, correct for extinction (as measured in Sect. 4) and finally convert this flux to the intrinsic $\mathrm{H} \alpha$ flux using the intrinsic line ratios. We assume Case B recombination $(10000 \mathrm{~K})$ giving a $\mathrm{H} \alpha$ to $\mathrm{H} \beta$ ratio of 2.85 . We then assume a star formation rate proportional to the $\mathrm{H} \alpha$ flux as $\Sigma_{\mathrm{SFR}}\left(M_{\odot} \mathrm{yr}^{-1}\right)=7.9 \times 10^{-42} L(\mathrm{H} \alpha)\left(\mathrm{erg} \mathrm{s}^{-1}\right)($ Kennicutt 1998). The measured star formation rates are given in Table 2. Again, we note that the emission features seen in Complex 1 are likely due to the overlapping HII region.

The star formation rates of the complexes are between 0.2 and $\sim 1.4 M_{\odot} \mathrm{yr}^{-1}$. These rates are extremely high, considering their relatively small sizes. Using the radius determined in the $F 555 \mathrm{~W}$ band (and assuming that the complexes are circular) the star formation rate per $\mathrm{kpc}^{2}\left(M_{\odot} \mathrm{yr}^{-1} \mathrm{kpc}^{-2}\right)$ is between 1 and 10. This is comparable to circumnuclear starbursts (Kennicutt 1998) and more than 10 times higher than the definition for a starburst put forward by Kennicutt et al. (2004).

These high star formation rates show that the complexes studied here can be thought of as localized starbursts (terminology from Efremov 2004). This coherent and intense form of star formation will significantly affect the surrounding ISM, both in distributing metals and in adding turbulence. These localized starbursts are expected to create a patchy metal distribution in the ISM, as observed in the Antennae (Fabbiano et al. 2004).

\section{Formation of the complexes}

\subsection{Insights from the HII}

Figure 15 shows the HST-WFPC2 continuum subtracted $\mathrm{H} \alpha$ images for 5 of the complexes in our sample which have extended $\mathrm{H} \alpha$ emission. The images are centered on the brightest cluster within each complex, which are marked with a red cross. Additionally, in two of the complexes (Complex 2 and
Complex Nuc 1) we indicate another cluster within the complex which appears to be the source of the ionization. The emission in Complex 6 (not shown) is concentrated on the brightest cluster, presumably due to the clusters very young age, which has not had time to create an extended ionization bubble.

In Complex 2, the brightest source appears to be older than other parts of the complex, and not responsible for the HII region. There is another bright source, which appears to be very young (6-9 Myr from Fig. 8). This bright young source is located near the center of the ionized region, suggesting that it is responsible for it. The photometry of the brightest source in Complex 2 gives an age of $\sim 25 \mathrm{Myr}$. At this age, no significant $\mathrm{H} \alpha$ emission is expected. If, on the other hand, the brightest cluster in this complex has a larger extinction than estimated in Sect. 4 then we would expect to see $\mathrm{H} \alpha$ emission, which is not present. The interpretation of this is that this complex contains clusters with different ages. This is highly reminiscent of the star cluster Hodge 301 in 30 Doradus. This cluster has an age of 25 Myr, much older than R136 (Grebel \& Chu 2000). Therefore multiple episodes of cluster formation within complexes may be a common feature.

As may have been expected, the complexes near the nucleus of NGC 4038 appear as the most complicated. Complex Nuc 2 is made up of multiple ionized regions, whereas Complex Nuc 1 also has a clear bubble like structure. The center of this bubble seems completely devoid of $\mathrm{H} \alpha$ and any optical source. The eastern region of the bubble contains two bright young clusters which may be the ionizing sources.

\subsection{Groupings of complexes}

In Fig. 16 we show the continuum subtracted HST-WFPC2 $\mathrm{H} \alpha$ image, along with the intensity contours. The sizes of the complexes in $\mathrm{H} \alpha$ and the $F 555 \mathrm{~W}$ bands is given in Table 2. The complexes are roughly the typical size of GMCs, and the masses derived are similar to that expected for GMCs of similar sizes ${ }^{2}$. The large ionized region surrounding Complexes 4 , 5 , and 6 has approximately the spatial size $(\sim 735 \mathrm{pc})$ where the two-point correlation function of young clusters drops suddenly (Zhang et al. 2001). This is also approximately the size of large GMCs or GMC complexes within the Antennae (Wilson et al. 2003). The common $\mathrm{H} \alpha$ envelope around this grouping of complexes and the presence of Wolf-Rayet features in each of the complexes, suggests that all of these complexes began to form at roughly the same time $(\Delta($ age $)<5-6 \mathrm{Myr})$. Complexes 4 and 5 also have extremely similar metallicities. Thus this grouping of complexes may have formed from a single GMC (or GMC complex) with multiple massive cores. Again we note that the presence of WR features and common $\mathrm{H}$ II envelope in Complexes 4, 5, and 6 only suggests that the most recent episode of star formation was coeval. But the lack of older clusters in these complexes suggests that the most recent episode of star formation was also the only or most significant bout of star formation.

\footnotetext{
${ }^{2}$ Using the data of spatially resolved clouds in The Antennae from Wilson et al. (2003), we find that $M_{\text {vir }}=0.024 \cdot R^{3.47}$, where $M$ and $R$ are in units of $M_{\odot}$ and pc respectively.
} 


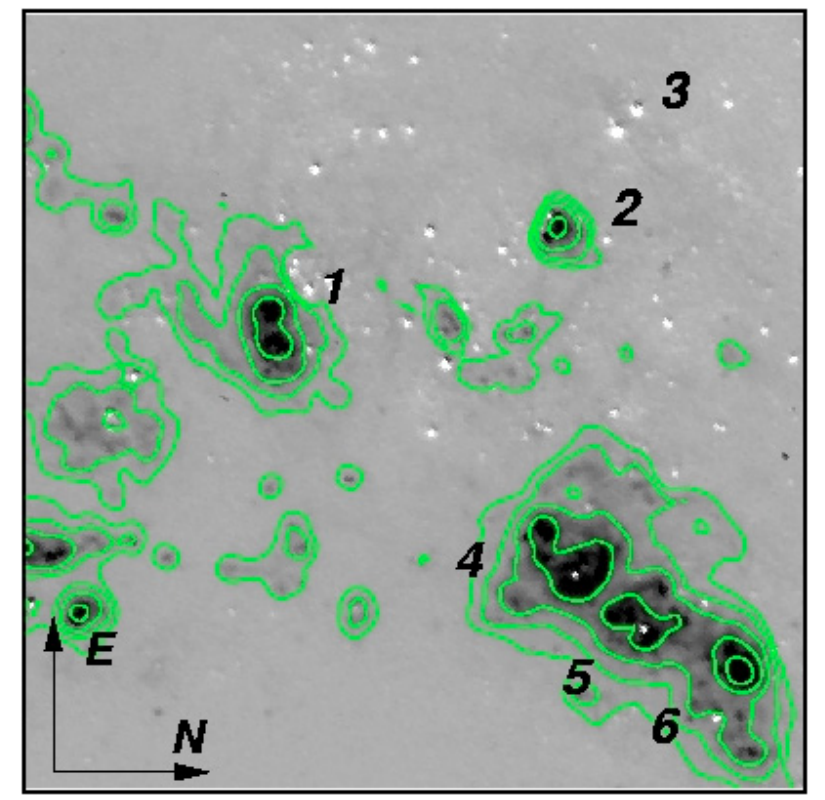

Fig. 16. The continuum subtracted $\mathrm{H} \alpha$ WFPC 2 image and contours. Note the large structure surrounding Complexes 4, 5, and 6. The shared envelope of excited gas surrounding these three complexes, along with the shared Wolf-Rayet features (see Fig. 12) may suggest a common age and a physical association. The reconstructed IFU image of $\mathrm{H} \beta$ intensity shows similar structure as the image shown here.

If we assume that this grouping of complexes formed out of the same GMC or GMC association, then it is interesting to compare the formation timescale and the sound-travel time. Complexes 4 and 5 are separated by $\sim 300 \mathrm{pc}$, while Complexes 5 and 6 are $\sim 350$ pc (note however that these are lower limits as these are projected distances). Assuming that the complexes have ages which are the same to within $3 \mathrm{Myr}$ (due to the presences of Wolf-Rayet features in all the complexes, as well derived from colour-colour diagrams, see Fig. 8), then to pass information of the formation of one to the next would require a speed of $>100 \mathrm{~km} \mathrm{~s}^{-1}$. Typical sound speeds in GMCs are below a few $\mathrm{km} \mathrm{s}^{-1}$ (Dyson \& Williams 1997). Thus, we see then that the triggered formation from one complex to the next is not likely, unless it was triggered by older, presently unobserved, star formation. One possibility for the coeval nature of the present star formation is an external perturbation, such as a large shock front, hit the parental GMC or GMC association at a very similar time throughout the entire cloud.

\subsection{Relation to giant molecular clouds}

Figure 17 shows the giant molecular cloud velocities in the overlap region of the NGC 4038/39 merger (Wilson et al. 2003). The dashed vertical lines represent the velocities of the complexes in Field 1. The match is extremely good, showing that the young complexes and the GMCs share the same general velocity distribution within this region. The main exception is the complex at $V=-153 \mathrm{~km} \mathrm{~s}^{-1}$ (with respect to the center of NGC 4038). This is the emission feature found in Complex 3. Near-IR spectroscopy of the two members

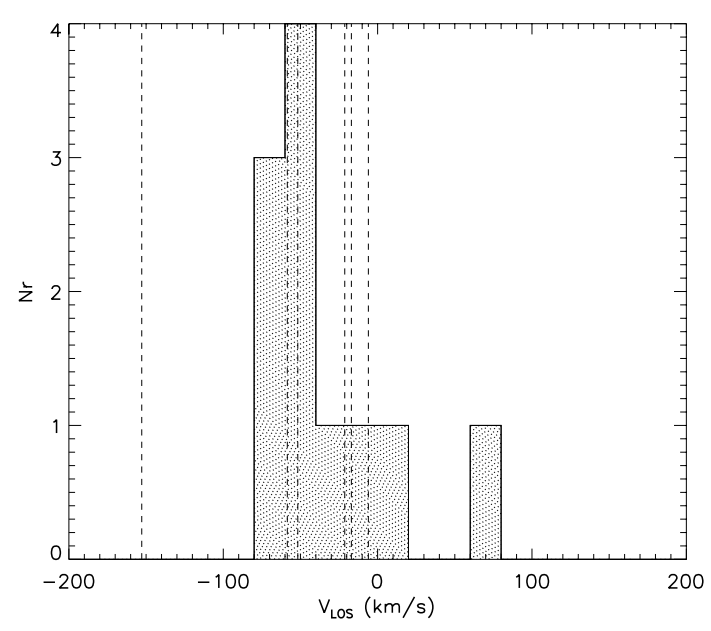

Fig. 17. Histogram of the line of sight velocities of GMCs in NGC $4038 / 39$ in the contact region $\left(\Delta 21^{\prime \prime}\right.$ in RA and $\Delta 40^{\prime \prime}$ in Dec centered on Field 1, taken from Wilson et al. 2003). The vertical dashed lines are the velocities of the complexes. The furthest line to the left is the emission feature observed in the direction of Complex 3 .

(Gilbert 2002) of Complex 3, show a continuum with no emission features. In particular the $\mathrm{Br} \gamma$ emission feature is absent, whereas it is expected to be present since we see relatively strong $\mathrm{H} \beta$ and $\mathrm{H} \gamma$ emission. Additionally, high resolution spectroscopy centered on the Complex 3 shows some hint of a shifted emission line $\sim 4$ arcsec from the center of the slit (Sabine Mengel, Priv. Comm.). The nature of the emission feature is unknown.

Upon closer examination, however, the complexes in Field 1 are located in a region devoid of molecular gas (see Fig. 4 in Wilson et al. 2003), although the detection limit of their survey was rather high at $\sim 5 \times 10^{6} M_{\odot}$. Thus they conclude that regions which have no detectable $\mathrm{CO}$ emission but contain young star clusters, either formed the clusters from lower masses clouds or the clusters present have destroyed their parent GMCs.

As shown in Sect. 5.1 the complexes in Field 1 have luminous masses between $\sim 0.6-5 \times 10^{6} M_{\odot}$, suggesting that their progenitor GMCs were greater than $5 \times 10^{6} M_{\odot}$. Thus, our data are consistent with the idea that the young clusters and complexes have destroyed their parent GMCs through their supernovae, stellar winds and ionizing photons.

\section{Stability of the complexes}

A number of recent studies (e.g. Kroupa 1998; Fellhauer \& Kroupa 2002) have suggested that the clusters within complexes, such as the ones studied here, will merge to form a single large star cluster in the center of the complex. This is expected to happen on timescales of a few $\times 10^{7}$ yr (Kroupa 1998). We therefore address the stability of the complexes in this section. We concentrate this analysis on Field 1, where the complicated dynamics that occurs within the center of merging galaxies should be less of a concern. 
As a simple initial assumption, we estimate the tidal radius of structures within a rotating disk. This can be estimated by

$r_{\mathrm{t}}=\left(\frac{G M_{\text {complex }}}{2 \cdot V_{\mathrm{G}}^{2}}\right)^{1 / 3} R_{\mathrm{G}}^{2 / 3}$

where $M_{\text {complex }}, V_{\mathrm{G}}$, and $R_{\mathrm{G}}$ are the mass of the complex, the circular velocity of the galaxy at that point, and the distance to the galactic center. Assuming a rotational velocity of $90 \mathrm{~km} \mathrm{~s}^{-1}$ (from Fig. 15 in Zhang et al. 2001) and a distance between 2 and $4 \mathrm{kpc}$ from the galactic center, we see that the tidal radii of complexes with masses between $10^{5}$ and $10^{6} M_{\odot}$ are between 50 and $150 \mathrm{pc}$. Additionally, given the steep velocity gradient at the position of Complexes 1, 4, and 5 the approximation given above will tend to overestimate the tidal radius. Comparison with Table 2 shows that most of the complexes are larger than their tidal radii, implying that a significant fraction of each complex is unbound.

Also, due to the rapid removal of gas from these systems (see Sect. 6) a substantial fraction of the mass of the complex, i.e. the gas left over from the star formation process, will be removed in a very short time. This will add to the amount of mass lost by the complexes. The inner regions of these complexes, however, may be less affected by these processes due to the higher cluster densities there, and merge to form a single massive object.

Due to the expected loss of clusters from these complexes, we expect them to contribute a large amount of their star clusters into the surrounding field. If merging does occur within the centers of the complexes, we expect that the resulting object will only contain a modest fraction of the total stellar mass of the progenitor complex.

\section{Conclusions}

We have presented the first results from a VLT-VIMOS/IFU survey of merging galaxies. Our targets were two fields within the Antennae galaxies, one in the overlap region and the other near the center of NGC 4038. In particular we have studied six star cluster complexes in the first region and two in the second region. We have estimated the extinction, metallicity, and velocity for each of these complexes using the emission lines $\mathrm{H} \gamma$, $\mathrm{H} \beta$, [OIII] $\lambda \lambda 4959,5007$, and $\mathrm{He} \mathrm{I}(\lambda$ 5876). The derived metallicities of the complexes are all above $0.5 Z_{\odot}$.

Assuming a common extinction value for all sources in each complex (see caveats to this assumption in Sect. 5.1), we are able to correct the HST photometry of the individual sources within each complex. The colours of the star clusters (once corrected for extinction) match the SSP models of Maraston (2005) extremely well. We determined the ages of the clusters using these colour-colour diagrams.

Complexes 4, 5, and 6 appear to be part of a larger structure approximately (in projection) $550 \mathrm{pc}$ in length. All three of these complexes show strong Wolf-Rayet features in their spectra, suggesting a common age $(\Delta($ age $)<4 \mathrm{Myr})$. This grouping of complexes is most notably visible in $\mathrm{H} \alpha$, where the complexes seem to share a common ionized envelope. This common ionized envelope, as well as the similarity in ages and metallicities of Complexes 4, 5, and 6 lead us to conclude that they were part of a single GMC or GMC complex, which fragmented into individual cluster complexes (e.g. a GMC association with multiple massive cores). The $\mathrm{H} \alpha$ spatial distributions are also hints that this collection of three complexes is destroying the surrounding molecular gas and dust. This is presumably what has happened in the eastern section of Field 1 where little gas and dust is seen.

The size of the large collection of complexes is approximately the same as the scale at which the two-point correlation function of young clusters drastically drops (i.e. at $\sim 735 \mathrm{pc}$ in $\mathrm{H} \alpha$ ). Thus, these groupings of complexes are likely to be the largest scale of correlated cluster formation. This scale corresponds to the size of large GMCs or GMC complexes, suggesting that a single GMC can form multiple complexes, each in turn containing many star clusters. Alternatively, the velocity differences between the complexes in this grouping $\left(\sim 65 \mathrm{~km} \mathrm{~s}^{-1}\right)$ may be an indication of high-velocity GMC collisions as the primary triggering mechanism, although the velocity difference does seem to follow the general velocity distribution in this region of the galaxy.

The youngest complexes in Field $1(<10 \mathrm{Myr})$ show rather small velocity dispersions (20-40 $\mathrm{km} \mathrm{s}^{-1}$ ) as measured through the $\mathrm{H} \beta$ and [OIII] $\lambda \lambda 44959,5007 \AA$ emission lines. These values can be understood as an expanding bubble, caused by the ionization and winds from massive stars and supernovae, that is expanding into a somewhat dense environment, i.e. the remnants of the progenitor GMC.

At least one complex in Field 1, Complex 2, shows evidence for a multiple aged population. The brightest cluster within the complex is not spatially coincident with the $\mathrm{H} \alpha$ emission in the region, and has a photometric age estimate of 7-15 Myr. Another bright cluster within the complex has a younger photometric age estimate, and is centered on the HII region. Thus we see that even a single complex can undergo multiple star formation episodes within $\sim 15 \mathrm{Myr}$. Complex Nuc 2 may also have a multiple-age cluster population, although the clusters lie parallel to the extinction vector (in colour-colour space) which suggests that they have similar ages but that there is variable amounts of extinction within the complex.

Using the dereddened $\mathrm{H} \beta$ emission line, we have estimated the star formation rates within the complexes. By normalizing the star formation rates by their areas, we see that these complexes, with star formation rates from 0.2 to $1.4 M_{\odot} / \mathrm{yr}$ are comparable to star forming regions in starburst galaxies. Thus we label these complexes as localized starbursts. This coherent and intense form of star formation may be responsible for the patchy distribution of metals observed in the ISM of the Antennae (Fabbiano et al. 2004).

The complexes in Field 1 have sizes similar to, or larger than, their tidal radii, estimated by assuming a smooth circular velocity profile of the galaxy. (Our tidal radii estimates are an upper limit, as the velocity profile of the galaxy, at the position of the complexes, is quite irregular.) Therefore we expect that these complexes will loose a significant amount of their mass 
(individual stars and clusters) into their surroundings. The central regions of the complexes, however, may be dense enough to allow a significant amount of merging between clusters, as predicted by Kroupa (1998).

Acknowledgements. This paper has used observations taken from the HST data archive, and has benefited from the NASA Extragalactic Database. We kindly thank Michele Cappellari for help implementing the PPXF technique and Mark Gieles, Yuri Efremov \& Linda Smith for useful discussions. We also thank Henny Lamers for detailed comments on earlier versions of the manuscript as well as for useful discussions. Finally, we thank the anonymous referee for helpful comments and suggestions.

\section{References}

Bastian, N., Gieles, M., Lamers, H. J. G. L. M., Scheepmaker, R. A., \& de Grijs, R. 2005a, A\&A, 431, 905

Bastian, N., Gieles, M., Efremov, Yu. N., \& Lamers, H. J. G. L. M. 2005b, in press [arXiv: astro-ph/0508110]

Bik, A., Lamers, H. J. G. L. M., Bastian, N., Panagia, N., \& Romaniello, M. 2003, A\&A, 397, 473

Comerón, F. 2001, A\&A, 365, 417

Cappellari, M., \& Emsellem, E. 2004, PASP, 116, 138

Dyson, J. E., \& Williams, D. A. 1997, in The Physics of the Interstellar Medium (Institute of Physics Publishing)

Edmunds, M. G., \& Pagel, B. E. J. 1984, MNRAS, 211, 507

Efremov, Yu. N. 2004, contribution to Gamow-100 [arXiv:astro-ph/0410702]

Efremov, Yu. N., Pustilnik, S. A., Kniazev, A. Y., et al. 2002, A\&A, 389,855

Elmegreen, B. G., \& Efremov, Y. N. 1996, ApJ, 466, 802

Elmegreen, B. G., Efremov, Yu. N., \& Larsen, S. S. 2000, ApJ, 535, 748

Fabbiano, G., Baldi, A., King, A. R., et al. 2004, ApJ, 605, L21

Fellhauer, M., \& Kroupa, P. 2002, AJ, 124, 2006

Fellhauer, M., \& Kroupa, P. 2005, MNRAS, 359, 223

Gilbert, A. 2002, Ph.D. Thesis, University of California, Berkely

Goudfrooij, P., Mack, J., Kissler-Patig, M., Meylan, G., \& Minniti, D. 2001, MNRAS, 322, 643

Grebel, E. K., \& Chu, Y.-H. 2000, AJ, 119, 787

Hibbard, J. E., van der Hulst, J. M., Barnes, J. E., \& Rich, R. M. 2001, AJ, 122, 2969

Kennicutt, R. C. 1998, ARA\&A, 36, 189

Kennicutt, R. C., Bresolin, F., \& Garnett, D. R. 2003, ApJ, 591, 801
Kennicutt, R. C., Lee, J. C., Funes, J. G., Sakei, S., \& Akiyama, S. 2004, in From 30 Doradus to Lyman break galaxies, ed. Richard de Grijs, \& Rosa M. González Delgado Starbursts, in press

Kroupa, P. 1998, MNRAS, 300, 200

Kroupa, P. 2001, MNRAS, 322, 231

Lamers, H. J. G. L. M., \& Cassinelli, J. P. 1999, in Introduction to Stellar Winds (Cambridge University Press)

Larsen, S. S., Efremov, Y. N., Elmegreen, B. G., et al. 2002, ApJ, 567, 896

Maraston, C., Bastian, N., Saglia, R., et al. 2004, A\&A, 416, 467

Maraston, C. 2005, MNRAS, 362, 799

Marlowe, A. T., Heckman, T. M., Wyse, R. F. G., \& Schommer, R. 1995, ApJ, 438, 563

Massey, P., \& Hunter, D. A. 1998, ApJ, 493, 180

Miller, B. W., Whitmore, B. C., Schweizer, F., \& Fall, S. M. 1997, AJ, 114,2381

Meyer, J.-P. 1985, ApJS, 57, 173

Norris, R. P. F. 2003, Ph.D. Thesis, University College London

Osterbrock, D. E. 1989, in Astrophysics of Gaseous Nebulae and Active Galactic Nuclei (Mill Valley: University Science Books)

Salpeter, E. E. 1995, ApJ, 121, 161

Savage, B. D., \& Mathis, J. S. 1979, ARA\&A, 17, 73

Schaller, G., Schaerer, D., Meynet, G., \& Maeder, A. 1992, A\&AS, 96, 269

Schaerer, D., Charbonnel, C., Meynet, G., Maeder, A., \& Schaller, G. 1993, A\&AS, 102, 339

Schweizer, F., Miller, B. W., Whitmore, B. C., \& Fall, S. M. 1996, AJ, 112,1839

Schweizer, F., \& Seitzer, P. 1998, AJ, 116, 2206

Smith, L. J., Norris, R. P. F., \& Crowther, P. A. 2002, MNRAS, 337, 1309

Stothers, R., \& Frogel, J. A. 1974, AJ, 79, 456

Tenorio-Tagle, G., Silich, S., Rogríguez González, A., \& Muñoz-Tuñón, C. 2005, ApJL, in press

Vacca, W. D., \& Conti P. S. 1992, ApJ, 401, 543

Van Bever, J., \& Vanbeveren, D. 2003, A\&A, 400, 63

Vazdekis, A. 1999, ApJ, 513, 224

Wilson, C. D., Scoville, N., Madden, S. C., \& Charmandaris, V. 2003, ApJ, 599, 1049

Whitmore, B. C., Heyer, I., \& Casertano, S. 1999, PASP, 111, 1559

Whitmore, B. C., Zhang, Q., Leitherer, C., et al. 1999, AJ, 118, 1551

Whitmore, B. C., Gilmore, D., Leitherer, C., et al. 2005, AJ, in press

Zepf, S. E., Ashman, K. M., English, J., Freeman, K. C., \& Sharples, R. M. 1999, AJ, 118, 752

Zhang, Q., Fall, M., \& Whitmore, B. C. 2001, ApJ, 561, 727 